\title{
A SHORT NOTE ON THE SUSCEPTIBILITY IN THE PARAMAGNETIC STATE FOR DISORDERED ITINERANT-ELECTRON METAMAGNETIC MATERIALS
}

\author{
M.A. GRADO-CAFFARO AND M. GRADO-CAFFARO \\ Julio Palacios 11, 28029 Madrid, Spain \\ (Received February 1, 1996; in final form March 29, 1996)
}

\begin{abstract}
A formulation for the paramagnetic susceptibility of a typical disordered itinerant-electron metamagnetic material is proposed by using a tight-binding approximation. Moreover, some considerations on the susceptibility tensor are exposed.
\end{abstract}

\section{INTRODUCTION}

Itinerant-electron metamagnetism has been observed in various intermetallic compounds as $\mathrm{RCo}_{2}, \mathrm{RMn}_{2}$, and $\mathrm{ThCo}_{5}$ [1] [2] [3] [4]. However, theoretical research on this subject has been scant so that it is necessary to develop accurate models to explain the involved phenomena. In the following, we will use a tight-binding approximation to calculate the magnetic susceptibility in the paramagnetic state for disordered itinerant-electron metamagnetic materials: In fact, such disordered materials have not been investigated either theoretically nor experimentally, so that our formulation constitutes a prediction based on solid prescriptions. Since a disordered material is isotropic, by assuming a diagonal susceptibility tensor, the three non-zero diagonal elements of this tensor are assumed to be the same.

\section{THEORY}

Firstly we formulate the following expression for the paramagnetic susceptibility of a typical itinerant-electron metamagnetic material [5]:

$\chi_{k k}=\gamma \sum_{i, j} \frac{\left\langle\psi_{0 i}\left|\hat{\mathrm{L}}_{k}\right| \psi_{1 j}><\psi_{1 j}\left|\hat{\mathrm{L}}_{k}\right| \psi_{0 i}>\right.}{\mathrm{E}_{1 j}-\mathrm{E}_{0 i}}(k=1,2,3)$

that is,

$$
\chi_{k k}=\gamma \sum_{i, j}\left|<\psi_{0 i}\right| \hat{\mathrm{L}}_{k}\left|\psi_{1 j}>\right|^{2}\left(\mathrm{E}_{1 j}-\mathrm{E}_{0 i}\right)^{-1}
$$


with $\chi_{11}=\chi_{22}=\chi_{33}(=\chi)$ by taking into account a susceptibility tensor $\overline{\bar{\chi}}=\chi \overline{\overline{1}}$. On the other hand, in expression (1) and (2) $\gamma$ is a real constant $(\gamma>0), \hat{\mathrm{L}} \equiv\left(\hat{\mathrm{L}}_{1}, \hat{\mathrm{L}}_{2}, \hat{\mathrm{L}}_{3}\right)$ is the angular momentum operator, and $\psi_{0 i}$ and $\psi_{1 j}$ are Schrödinger eigenfunctions such that $\left.\hat{\mathrm{H}}\left|\psi_{0 i}>=\mathrm{E}_{0 i}\right| \psi_{0 i}\right\rangle, \hat{\mathrm{H}}\left|\psi_{1 j}>=\mathrm{E}_{1 \mathrm{j}}\right| \psi_{1 j}>$. We assume two bands labeled 0 and 1, which correspond to the spin-up and spin-down bands, respectively, so that $\psi_{0 i}$ refers to the spin-up band and $\psi_{1 j}$ refers to the spin-down band. Also, $i$ and $j$ label the states within the bands. Next we consider the following orthonormal tight-binding basis:

$\left|\psi_{0 i}>=\sum_{\alpha}<\psi_{0 \alpha}\right| \psi_{0 i}>\mid \psi_{0 \alpha}>$

$\left|\psi_{1 i}>=\sum_{\alpha}<\psi_{1 \alpha}\right| \psi_{1 i}>\left|\psi_{1 \alpha}\right\rangle$

so that we get:

$$
\begin{aligned}
& \sum_{\beta}\left|\psi_{0 \beta}><\psi_{0 \beta}\right|=\sum_{\alpha}\left|\psi_{0 \alpha}><\psi_{0 \alpha}\right| \\
& \sum_{\beta}\left|\psi_{1 \beta}><\psi_{1 \beta}\right|=\sum_{\alpha}\left|\psi_{1 \alpha}><\psi_{1 \alpha}\right|
\end{aligned}
$$

In addition, we have:

$\chi \approx c \gamma\left\{\sum_{i, j}\left[<\psi_{1 j}\left|\hat{\mathrm{L}}_{k}\right| \psi_{0 i}>\left(\int_{0}^{\infty}<\psi_{0 i}\left|e^{u-u \hat{\mathrm{H}}} \hat{\mathrm{L}}_{k} e^{-u \hat{\mathrm{H}}_{\mid}}\right| \psi_{1 j}>\cdot d u\right)\right]\right\}$

where $\mathrm{c}$ is a real constant (c >0). Finally, expression (7) can be transformed by using the basis formed by (3) and (4) so that we obtain:

$\chi \approx c \gamma\left\{\sum_{i, j}\left[<\psi_{1 j}\left|\hat{\mathrm{L}}_{k}\right| \psi_{0 i}>\left(\int_{0}^{\infty}<\psi_{0 i}\left|\hat{\mathrm{L}}_{k}(-u)^{\dagger}\right| \psi_{1 j}>. d u\right)\right]\right\}$

where $\hat{\mathrm{L}}_{\mathrm{k}}(\mathrm{u}) \equiv \mathrm{e}^{\mathrm{u} \hat{\mathrm{H}}} \hat{\mathrm{L}}_{\mathrm{k}} \mathrm{e}^{-\mathrm{uH}}$.

\section{CONCLUDING REMARKS}

By using an orthonormal tight-binding basis, we have obtained an expression for the paramagnetic susceptibility of a typical disordered itinerant-electron metamagnetic material. This relationship becomes useful to understand certain aspects related to the Stoner model [6] [7]. Our theory can be linked with new mechanisms for itinerant-electron metamagnetism and we can claim that this theoretical 
formulation is related to the coupling between electrons and atom clusters. On the other hand, disorder constitutes an ingredient that introduces serious difficulties in the above context.

\section{REFERENCES}

1. R. Lemaire. Cobalt 33, 201 (1966).

2. G. Petrich, R.L. Mössbauer. Phys. Lett. A 26, 403 (1968).

3. T. Goto, K. Fukamichi, T. Sakakibara, H. Komatsu. Solid State Communications 72, 945 (1989).

4. F. Givord, J.S. Shah. C.R. Acad. Sc. Paris B 274, 923 (1972).

5. M.A. Grado-Caffaro, M. Grado-Caffaro (unpublished).

6. M.A. Grado-Caffaro, M. Grado-Caffaro. Act. Pass. Electronic Comp. 17, 177-178 (1994).

7. M.A. Grado-Caffaro, M. Grado-Caffaro. (unpublished). 

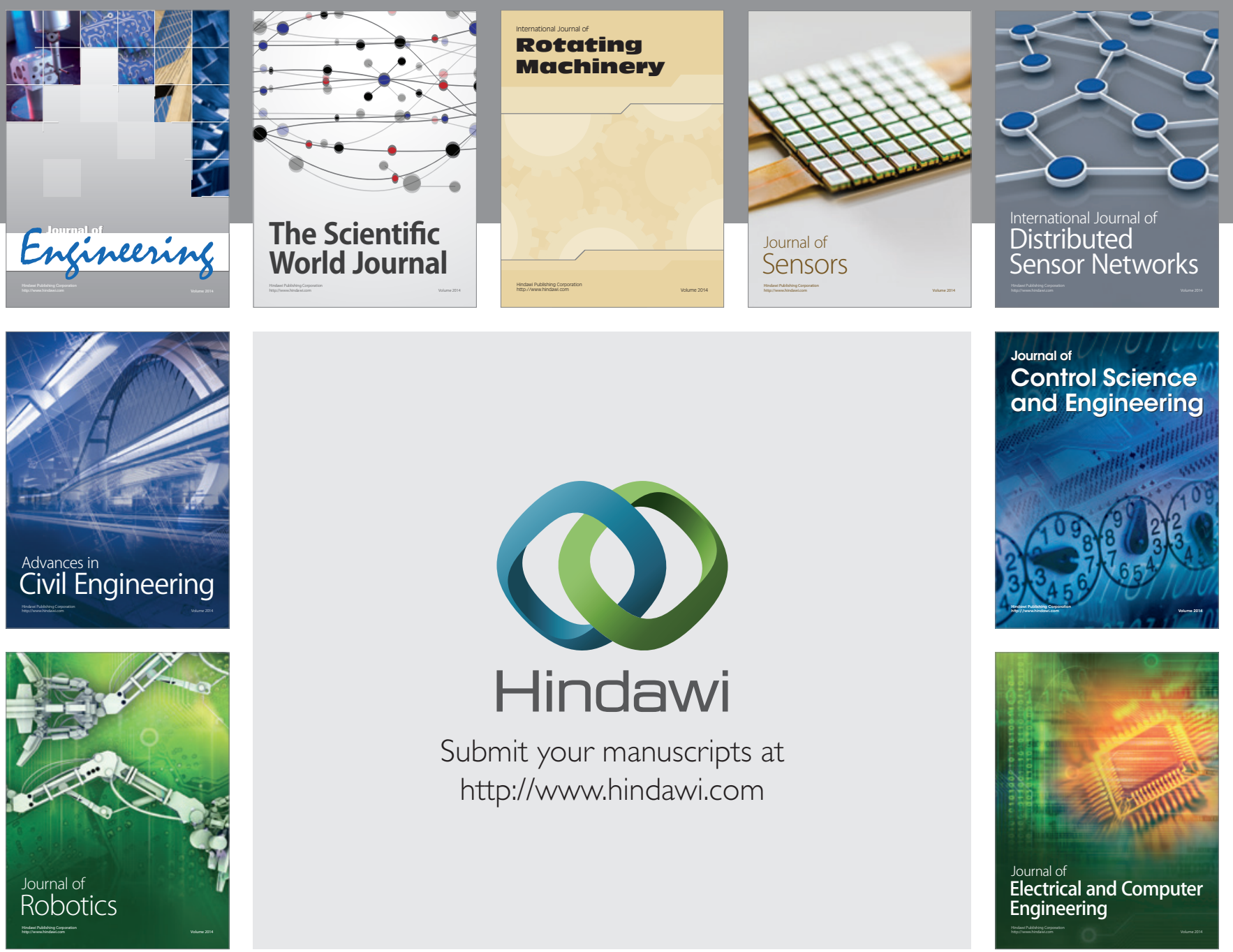

Submit your manuscripts at

http://www.hindawi.com
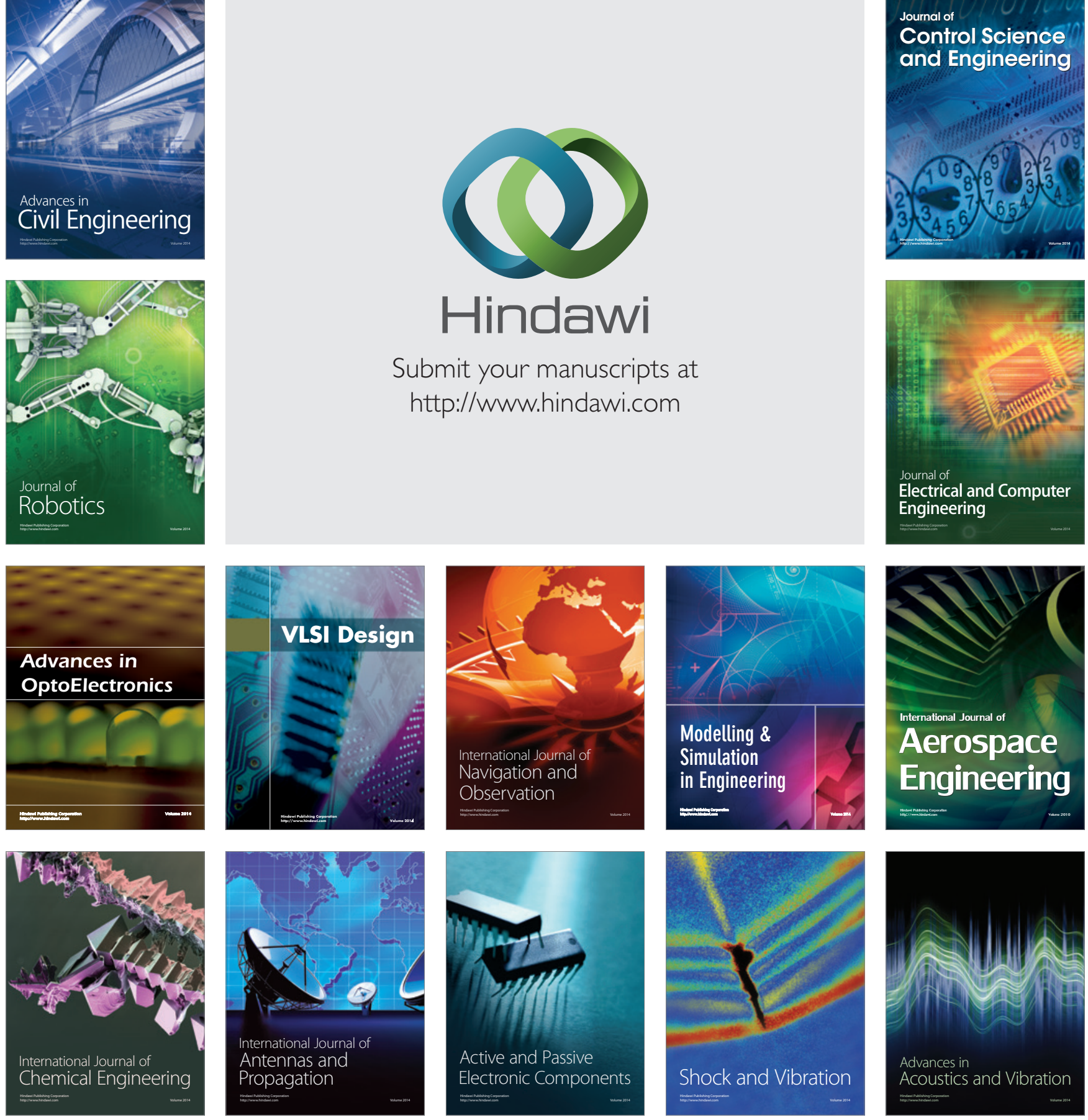